\title{
Phylogeny and evolution of plant macrophage migration inhibitory factor/D-dopachrome tautomerase-like proteins
}

\author{
Ralph Panstruga ${ }^{*}$, Kira Baumgarten ${ }^{1}$ and Jürgen Bernhagen ${ }^{2}$
}

\begin{abstract}
Background: The human (Homo sapiens) chemokine-like protein macrophage migration inhibitory factor (HsMIF) is a pivotal mediator of inflammatory, infectious and immune diseases including septic shock, colitis, malaria, rheumatoid arthritis, and atherosclerosis, as well as tumorigenesis. HsMIF has been found to exhibit several sequential and threedimensional sequence motifs that in addition to its receptor binding sites include catalytic sites for oxidoreductase and tautomerase activity, which provide this $12.5 \mathrm{kDa}$ protein with a remarkable functional complexity. A human MIF paralog, D-dopachrome tautomerase (HsDDT), has been identified, but its physiological relevance is incompletely understood. MIF/ DDT-like proteins have been described in animals, protists and bacteria. Although based on sequence data banks the presence of MIF/DDT-like proteins has also been recognized in the model plant species Arabidopsis thaliana, details on these plant proteins have not been reported.

Results: To broaden the understanding of the biological role of these proteins across kingdoms we performed a comprehensive in silico analysis of plant MIF/DDT-like (MDL) genes/proteins. We found that the A. thaliana genome harbors three MDL genes, of which two are chiefly constitutively expressed in aerial plant organs, while the third gene shows stress-inducible transcript accumulation. The product of the latter gene likely localizes to peroxisomes. Structure prediction suggests that all three Arabidopsis proteins resemble the secondary and tertiary structure of human MIF. MIFlike proteins are found in all species across the plant kingdom, with an increasing family complexity towards evolutionarily advanced plant taxa. Plant MDL proteins are predicted to lack oxidoreductase activity, but possibly share tautomerase activity with human MIF/DDT.
\end{abstract}

Conclusions: Peroxisome localization seems to be a specific feature of a subset of MIF/DDT orthologs found in dicotyledonous plant species, which together with its stress-inducible gene expression might point to convergent evolution in higher plants and vertebrates towards neofunctionalization of MIF/MDL proteins in stress response pathways including innate immunity.

Keywords: Arabidopsis thaliana, Co-expression, D-dopachrome tautomerase, Macrophage migration inhibitory factor, Neofunctionalization, Oxidoreductase, Peroxisomes, Phylogeny

\section{Background}

In Homo sapiens, the protein family of chemotactic cytokines, i.e. chemokines, functions as traffic coordinators for the innate defense system, but it also orchestrates lymphocyte trafficking and homeostatic cell homing in the human body. Thus, chemokines represent a fundamental component in innate and adaptive immunity

\footnotetext{
* Correspondence: panstruga@bio1.rwth-aachen.de

${ }^{1}$ RWTH Aachen University, Institute of Biology I, Unit of Plant Molecular Cell Biology, Worringerweg 1, 52074 Aachen, Germany

Full list of author information is available at the end of the article
}

[1-9]. However, chemokines have also been subject to various mimicry mechanisms exploited by viruses and parasites. Macrophage migration inhibitory factor (MIF) is an evolutionarily ancient protein that is best known for its functions as an immune and inflammatory factor and that was more recently recognized to have chemokine-like properties to regulate a plethora of processes in the biology and pathophysiology of humans $[10,11]$. MIF is a prototypical member of the growing functional family of CLF (chemokine-like function) chemokines that share with classical chemokines chemokine 
receptor-mediated chemotaxis and cell recruitment activities, but that do not possess the canonical $\mathrm{N}$-terminal cysteine residues or chemokine-fold [12-15]. Human MIF (HsMIF) has 114 amino acids, forms a trimer in the $\mathrm{X}$-ray structure $[10,16]$, and is a required upstream component of human innate and adaptive immunity, but it is also overexpressed in various human diseases [10]. If dysregulated, $H s$ MIF is a pivotal mediator of inflammatory, infectious and immune diseases including septic shock, colitis, malaria, rheumatoid arthritis, and atherosclerosis, as well as in several tumors $[14,17]$. In fact, $H s$ MIF was discovered already 50 years ago, but it took until the early 1990s and mid-2000s until the MIF protein was characterized and the MIF receptors were identified, respectively [18-21]. MIF functions are mediated through three receptor proteins: on the one hand, MIF signals through the type II receptor protein CD74/invariant chain, but on the other hand it also serves as a non-cognate ligand for the classical CXC chemokine receptors CXCR2 and CXCR4 $[19,20]$.

The structural interfaces governing MIF/receptor interactions have partly been unraveled, but important mechanistic details of its structure-activity characteristics, cell- and environment-specific receptor usage, cross-reactivity, interplay with bona fide ligands, or receptor complexes are unclear [14]. Moreover, MIF is abundantly expressed in the cytosol of numerous cells and features two evolutionarily conserved catalytic sites, i.e. a dopachrome tautomerase and a thiol-protein oxidoreductase (TPOR) activity, implying links to MIF's role in cell cycle regulation $[10,22]$. The catalytic activities have been suggested to localize to a threedimensional proline-2-containing tautomerase site at the $\mathrm{N}$-terminus and a Cys-Xaa-Xaa-Cys motif-spanning sequence at amino acids 57-60, respectively. Both catalytic $H s$ MIF activities can be readily measured in vitro, but physiologic substrates have been elusive and the functional role of the activities in vivo is unclear. Strikingly, HsMIF and its human paralog MIF-2/D-DT (D-dopachrome tautomerase, hereafter $H s \mathrm{DDT}$ ) are found across kingdoms with expression verified in mammals, Хепориs laevis, Caenorhabditis elegans, Bifidobacterium longum, Clostridium acetobutylicum, or unicellular parasites such as Brugia malayi. While co-expression of DDT and MIF has been observed in some species, functional evidence on DDT has been scarce. DDT shares with MIF an exacerbating role in endotoxic shock and models of melanoma, non-small cell lung carcinoma, and renal tumorigenesis, and mimics the cardioprotective effect of MIF in a mouse model of ischemia/reperfusion injury of the heart [23-26]. In contrast, the effects of DDT on cell survival and apoptosis are complex and the receptor mechanisms conveying DDT activity are unclear at the molecular level. Also, a combined functional analysis of both MIF proteins in other organisms has not yet been undertaken.

Arabidopsis thaliana is a dicotyledonous plant species and is arguably the best-studied model plant [27]. Although its genome sequence has been resolved more than a decade ago [28], approximately half of its ca. 30.000 genes remain functionally unknown or are annotated only on the basis of static analyses such as protein motifs or similarities [29]. The uncharacterized genes also include apparent MIF-like genes, whose existence in the Arabidopsis genome has been previously noted $[30,31]$. However, the genes have not yet attained any attention by the plant or cytokine/chemokine community, and accordingly neither the genes nor the respective gene products have been characterized to date.

To further broaden the understanding of the role of MIF-like proteins across kingdoms we performed a comprehensive in silico analysis of plant MIF/DDT-like (MDL) genes/proteins with an emphasis on the reference species Arabidopsis thaliana. We deployed several analysis tools to unravel the copy number, gene structure, expression profile and predicted subcellular localization of the Arabidopsis MDL genes/proteins. We extended the study by sequence alignment and phylogenetic analysis including a broad range of MDL sequences across the entire plant kingdom.

\section{Results}

\section{Arabidopsis thaliana encodes three MIF-like proteins}

We performed BLASTP searches against the predicted proteome of the dicotyledonous reference species Arabidopsis thaliana (TAIR, http://www.arabidopsis.org/) using human MIF (GenBank accession number P14174) as a query sequence. This analysis revealed three hits with moderate sequence similarity to $H s \mathrm{MIF}$, comprising proteins At5g57170 (E value 3e-12, 33\% identity), At5g01650 (E value 2e-12, 30\% identity) and At3g51660 (E value 9e-7, 28\% identity). The three proteins are of similar length as human MIF and DDT (115, 115 and 112 amino acids for the three Arabidopsis proteins versus 115 amino acids for $H s$ MIF and 118 for $H s$ DDT); accordingly, their calculated molecular weight is within a comparable range (ca. 11.3-12.7 kDa; Table 1). These values refer to the conceptual full-length proteins as predicted from the corresponding cDNA sequences. Human MIF and DDT are known to undergo proteolytic processing of the N-terminal methionine and thus in its mature form comprise only 114 and 117 amino acids, respectively $[25,32]$. The sequence similarity to human MIF and DDT extends nearly along the entire protein, with 14 invariant amino acid residues interspersed, except for the very C-terminus, which shows little sequence conservation between the five aligned proteins (Figure 1). The 14 invariant amino acids include a proline at position 2 
Table 1 Features of AtMDL proteins in comparison to HsMIF and HsDDT

\begin{tabular}{llllll}
\hline & HsMIF & HsDDT & At5g57170 (AtMDL1) & At5g01650 (AtMDL2) & At3g51660 (AtMDL3) \\
\hline Number of amino acids & 115 & 118 & 115 & 115 & 112 \\
Molecular mass $^{\mathrm{a}}$ & 12476 & 12712 & 12324 & 12111 & 12210 \\
Isoelectric point (pl) $^{\mathrm{a}}$ & 7.73 & 6.72 & 4.96 & 6.17 & 8.82 \\
InterProScan domains & IPR001398 & IPR001398 & IPR001398 & IPR001398 & IPR001398 \\
& IPR014347 & IPR014347 & IPR014347 & IPR014347 & IPR014347 \\
& IPR019829 & IPR019829 & & & C-terminal PTS1 $^{c}$ \\
\hline
\end{tabular}

afull-length protein based on calculation with ExPASy (http://web.expasy.org/compute_pi/); note that in most cases analyzed so far the N-terminal methionine is post-translationally removed by proteolytic processing).

${ }^{\mathrm{b}}$ nuclear export sequence.

cperoxisome targeting sequence 1 .

$\left(\mathrm{P}^{2}\right.$; note that numbering of amino acids in this study refers to the conceptual full-length sequences, disregarding the proteolytic processing of the N-terminal methionine), which is essential for the tautomerase activity of $H s$ MIF [33]. By contrast, the two cysteine residues $\left(C^{57}\right.$ and $\left.C^{60}\right)$ that form the TPOR motif and are crucial for the oxidoreductase activity in HsMIF [22,34] are not preserved in the Arabidopsis proteins (Figure 1). The Arabidopsis proteins also lack the so-called pseudo ELR motif (Figure 1). This sequence motif, which is comprised of the amino acids glutamate (E), leucine (L) and arginine (R), is found in a variety of chemokines. It is critical for receptor binding and essential for chemotactic activity of $\mathrm{ELR}^{+} \mathrm{CXC}$ chemokines [35]. In HsMIF, it is present in a non-canonical manner ('pseudo' ELR motif), constituted by non-adjacent residues in neighboring loops but with identical parallel spacing as in the authentic ELR motif [36].

Analysis with InterProScan 5 (http://www.ebi.ac.uk/ interpro/) indicated the presence of characteristic MIF (IPR001398) and tautomerase (IPR014347) domains as the sole recognizable features in the three Arabidopsis proteins (Table 1). Accordingly, we named these Arabidopsis MIF/DDT-like (MDL) polypeptides AtMDL1 (At5g57170), AtMDL2 (At5g01650) and AtMDL3 (At3g51660), although the IPR019829 motif (MIF conserved site; consensus sequence [DE]-P-[CLV]-[APT]$\mathrm{x}(3)-[\mathrm{LIVM}]-\mathrm{x}-\mathrm{S}-[\mathrm{IS}]-[\mathrm{GT}]-\mathrm{x}$-[LIVM]-[GST]), which is located at amino acids 55-67 in $H s$ MIF and $H s$ DDT and corresponds to the catalytic TPOR site in HsMIF, is missing in the three Arabidopsis proteins (Table 1,

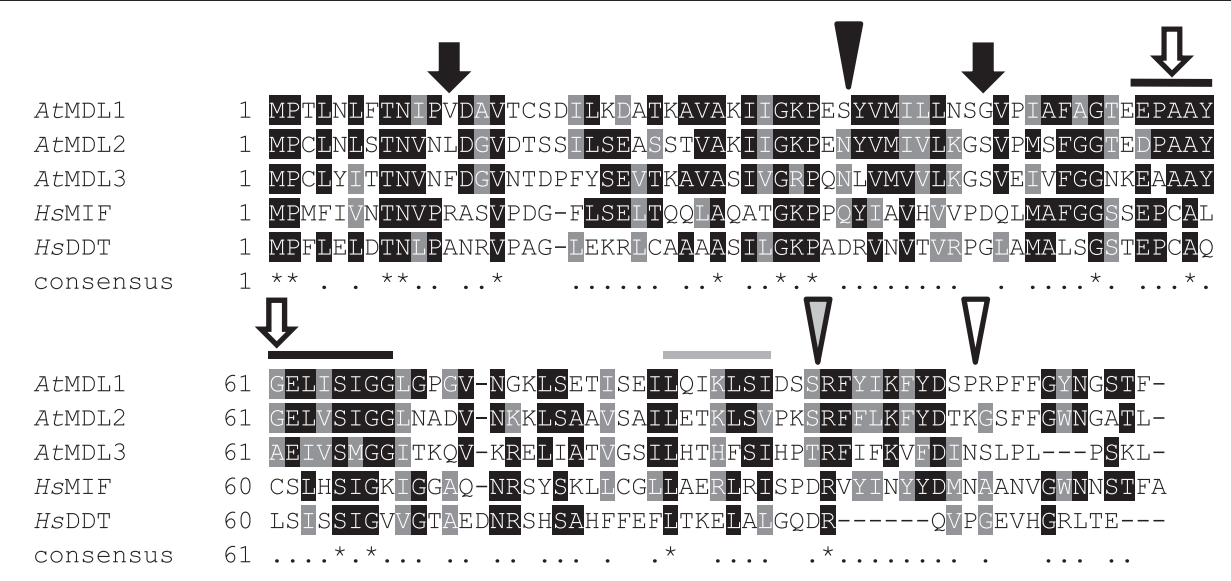

Figure 1 Multiple sequence alignment of AtMDLs, HsMIF and HsDDT. Amino acid sequences of AtMDL1 (AAO42959), AtMDL2 (NP_195785), AtMDL3 (AEE78824), HsMIF (P14174) and HsDDT (P30046) were aligned with ClustalW2 (http://www.ebi.ac.uk/Tools/msa/clustalw2/) using standard parameters. Subsequently, the alignment was shaded with BoxShade 3.21 (http://www.ch.embnet.org/software/BOX_form.html) using default settings. A black shade indicates identical amino acids; a grey shade denotes similar amino acids. In the consensus line, asterisks specify invariant amino acids and dots conserved amino acids with similar biophysical properties at a given position. The black triangle highlights the relative position of an intron found in all five genes, the white triangle the relative position of an intron present in AtMDL1 and AtMDL2 and the grey triangle the relative position of an intron found in HsMIF and HsDDT. The white arrows denote the position of $\mathrm{C}^{57}$ and $\mathrm{C}^{60}$ in $\mathrm{HsMIF}_{\mathrm{S}}$, the black bar above part of the sequence indicates the location of InterProScan domain IPR019829 (MIF, conserved site) in HsMIF and HsDDT, which overlaps with the TPOR motif. The black arrows point at residues $R^{12}$ and $D^{45}$ in HSMIF that form the pseudo-ELR motif and the grey bar above part of the sequence signifies the position of the predicted NES in AtMDL1 and HsMIF. 
Figure 1). The three AtMDL proteins exhibit considerable pairwise sequence similarity with AtMDL1 and AtMDL2, showing a higher degree of relatedness to each other than to AtMDL3 (AtMDL1-AtMDL2: 60\% identity, 75\% similarity; AtMDL1-AtMDL3: 40\% identity, 57\% similarity; AtMDL2-AtMDL3: 50\% identity, $69 \%$ similarity). This is somewhat higher than the kinship of HsMIF and HsDDT, which exhibit 37\% identity and $52 \%$ similarity. Despite the high overall degree of sequence relationship, the calculated isoelectric point (pI) of the three Arabidopsis MDL proteins differs greatly, with a pI of 5.0 for AtMDL1, 6.2 for AtMDL2 and 8.8 for AtMDL3, as compared to a pI of 7.7 for $H s$ MIF and 6.7 for $H s$ DDT (Table 1). The three Arabidopsis MDL proteins and their respective genes are largely uncharacterized since according to our literature searches no studies with functional data are available to date.

\section{Genomic organization and distribution of AtMDL genes}

For AtMDL1 and AtMDL2, the Arabidopsis reference database TAIR (The Arabidopsis Information Resource; http://www.arabidopsis.org/) lists two distinctive gene models each (At5g57170.1/At5g57170.2 and At5g01650.1/ At5g01650.2, respectively). These models differ in the number of exons/introns and the localization of splice sites, resulting in different predicted transcripts, which suggests the potential occurrence of alternative splicing events at the transcript level. However, BLAST searches against Arabidopsis expressed sequence tags (ESTs) at the NCBI database solely support gene models At5g57170.1 and At5g01650.1, suggesting that the two other models (At5g57170.2 and At5g01650.2) may either rely on faulty genome annotation or may represent rare splicing variants. In the following, we therefore only considered the conceptual amino acid sequences that are based on gene models At5g57170.1 and At5g01650.1.

AtMDL1 and AtMDL2 are characterized by the presence of two introns, while AtMDL3 has a single intron. The former is similar to the situation of $H s M I F$, which also harbors two introns, whereas the human $D D T$ gene has three introns, of which the first is located outside the coding sequence in the $5^{\prime}$-untranslated region. Notably, the relative position of the first intron in At $M D L 1$, AtMDL2 and HsMIF, the second intron in HsDDT and the sole intron in AtMDL3 is precisely preserved (Figure 1). This finding indicates common ancestry of the plant and human MIF/DDT genes and suggests that at least part of the genomic organization of the MIF/DDT genes has been retained since the separation of the plant and animal lineages ca. 1.6 billion years ago [37]. The relative positions of the second introns are also conserved for AtMDL1 and AtMDL2 on the one hand and HsMIF and HsDDT on the other hand, indicating the acquisition of lineage-specific introns later during evolution (Figure 1).
The Arabidopsis thaliana genome has been shaped by a whole genome duplication event, which resulted in extended yet reshuffled blocks of tandem repeated genomic regions that exhibit large-scale conservation in the number, order and orientation of genes [38]. Interrogation of the Plant Genome Duplication Database (http://chibba. agtec.uga.edu/duplication/) revealed that none of the AtMDL genes has a recognizable counterpart as the result of intragenomic duplication. This finding suggests that the diversification of the AtMDL genes occurred prior to the whole genome duplication event, which has been estimated to have occurred ca. 38 million years ago [39].

\section{Structure prediction of AtMDL proteins}

The three-dimensional (3D) structure of $H s$ MIF has been initially resolved by X-ray crystallography at $2.6 \AA$ resolution [16]. HsMIF crystallizes as a trimer, while solution analyses have produced variable results about the oligomerization state of $H s \mathrm{MIF}$, ranging from monomer to dimer or trimer species depending on the protein concentration and method applied [13]. The HsMIF monomer consists of two antiparallel $\alpha$-helices that pack against a four-stranded $\beta$-sheet. A 3D topology with similar structural elements was subsequently determined for $H s \mathrm{DDT}$ on the basis of X-ray crystallography at a resolution of $1.54 \AA$ [40]. We used the fold recognition server Phyre $^{2}$ (Protein Homology/analogY Recognition Engine 2, http://www.sbg.bio.ic.ac.uk/phyre2/html/page.cgi?id=index) to predict in silico the 3D structure of the three AtMDL monomers. This analysis, which is based on homology modelling, revealed a surprisingly well conserved tentative 3D structure of the Arabidopsis MDL proteins in comparison to HsMIF (Figure 2), despite the limited similarity of these proteins at the level of the primary amino acid sequence (ca. 30\% identity - see above). The noted architectural conservation is reminiscent of the high degree of 3D similarity between mammalian MIF/DDT proteins and tautomerase-active homologs found in protists (e.g. Leishmania major MIF, [41]) and bacteria (e.g. 4oxalocrotonate tautomerase (4-OT) and 5-(carboxymethyl)-2-hydroxymuconate isomerase (CHMI; [33]). Further support for similar structures of HsMIF and the AtMDL proteins is provided by a second structure prediction algorithm (QUARK), which allows $a b$ initio calculation of protein $3 \mathrm{D}$ structures. Although the 3D structure of HsMIF monomer predicted with QUARK differs from the experimentally determined structure (in contrast to the X-ray based structure the two $\alpha$-helices flank the $\beta$-sheets), QUARK calculated similar 3D structures for $H s \mathrm{MIF}$ and the three AtMDL proteins (Additional file 1: Figure S1). Together these findings further strengthen the notion that the AtMDL proteins are bona fide coorthologs of $H s \mathrm{MIF}$ and $H s \mathrm{DDT}$ that may share functional conservation with respect to core biochemical activities of 

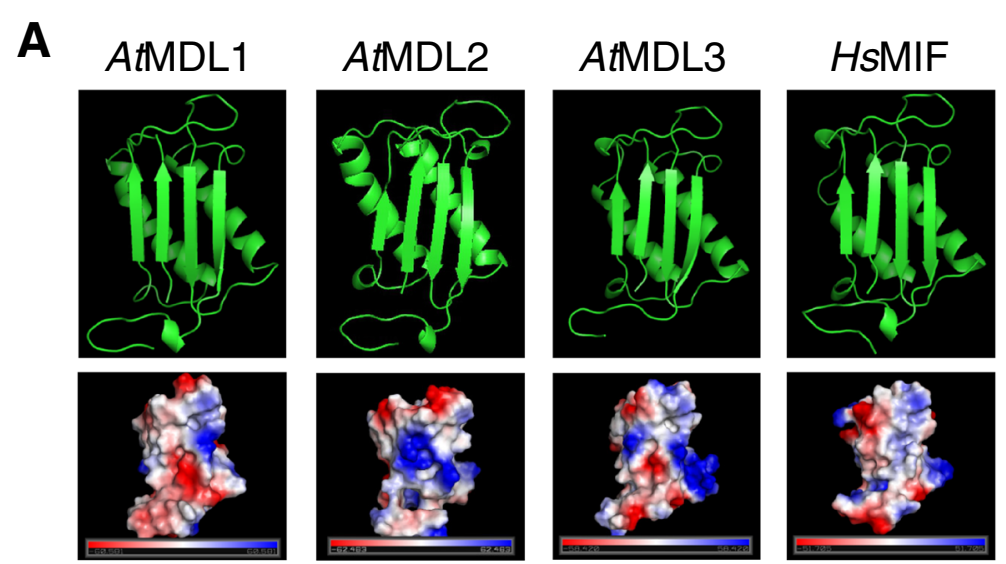

\section{B}
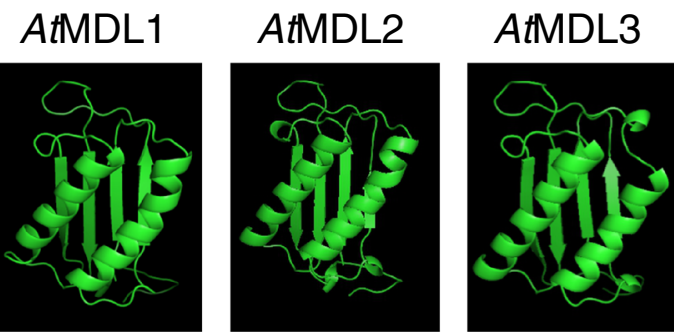

HsMIF
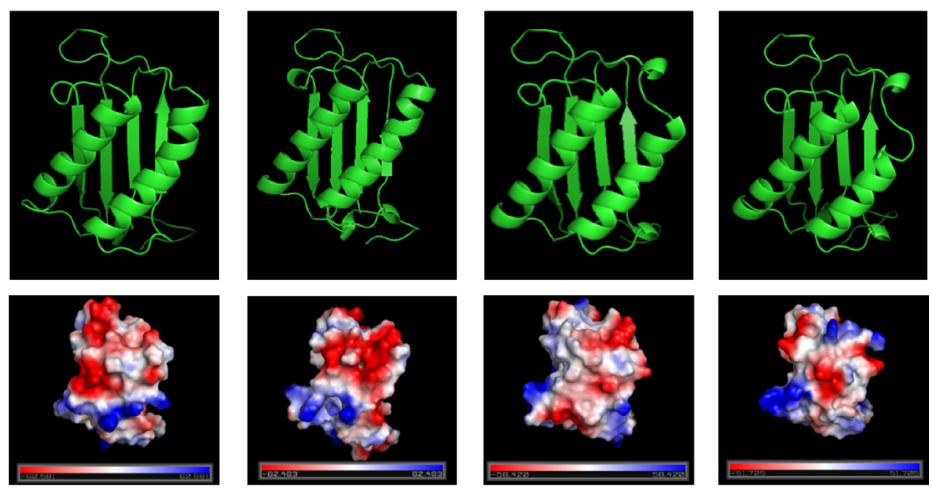

Figure 2 Predicted AtMDL 3D structures. Amino acid sequences of the AtMDL proteins were subjected to analysis via the PHYRE ${ }^{2}$ Fold Recognition server (http://www.sbg.bio.ic.ac.uk/phyre2/html/page.cgi?id=index) and rendered with PyMOL (http://www.pymol.org/). The predicted 3D structures (ribbon models and electrostatic surface potential) of AtMDL1, AtMDL2 and AtMDL3 are visualized in comparison to the known X-ray-resolved 3D structure of HsMIF. In the upper panels, green arrows symbolize $\beta$-sheets and green coils a-helices. In the lower panels, red color indicates an excess of negative and blue color an excess of positive charges near the surface, while white color specifies neutral regions. For simplicity, the structure of the monomer is shown only. A View with the four-stranded $\beta$-sheet in front. B View with the two antiparallel ahelices in front.

this protein class. We further visualized the electrostatic surface potential of these proteins to find out whether the noted differences in the $\mathrm{pI}$ of MIF/MDL proteins (Table 1) is reflected by prominent alterations in surface charge distribution. Since this was not the case, we speculate that the amino acids causing the charge differences are either not surface-exposed and/or distributed to such an extent that differences become unrecognizable.

\section{Subcellular localization of AtMDL proteins}

Using a panel of prediction servers, we inspected the three AtMDLs in silico for the presence of canonical targeting signals that could provide first hints on their subcellular localization. All three proteins lack a number of analyzed targeting signals for dedicated subcellular localization, including N-terminal secretion signals (SignalP 4.1, http:// www.cbs.dtu.dk/services/SignalP/), transmembrane domains (TMHMM 2.0, http://www.cbs.dtu.dk/services/
TMHMM/), transit peptides for mitochondrial (Mitoprot, http://ihg.gsf.de/ihg/mitoprot.html) or chloroplast (ChloroP 1.1, http://www.cbs.dtu.dk/services/ChloroP/) localization and nuclear import signals (NLStradamus, http://www. moseslab.csb.utoronto.ca/NLStradamus/). Lack of these sequence motif features is shared by $H s \mathrm{MIF}$ and $H s \mathrm{DDT}$, which are secreted by non-conventional pathways that do not require ER/Golgi transit [42,43]. However, AtMDL1 has a predicted leucine-rich nuclear export signal (NetNES 1.1, http://www.cbs.dtu.dk/services/NetNES/) at amino acid positions 85-91 and AtMDL3 possesses a predicted Cterminal peroxisomal targeting sequence (PTS1 predictor; http://mendel.imp.ac.at/mendeljsp/sat/pts1/PTS1predic tor.jsp) (Table 1). Notably, the predicted NES is shared by $H s \mathrm{MIF}$, where this putative signal occurs at the corresponding relative position (amino acid positions 83-90; Table 1).

Two of the three AtMDLs have been previously identified in shot-gun proteomic studies of Arabidopsis organelles. 
AtMDL1 was found as a stromal protein in the chloroplast proteome [44,45] and AtMDL3 was discovered as a peroxisomal protein [46]. The latter localization is consistent with the presence of a C-terminal PTS1 localization signal in AtMDL3 (Table 1) and also with fluorophore-based subcellular localization studies [47], whereas the experimentally found stromal localization of AtMDL1 is not supported by a recognizable chloroplast-targeting sequence. Nevertheless, further experimental evidence is needed to substantiate the predicted subcellular localization of the AtMDL proteins.

\section{Expression profiles of $A t M D L$ genes}

We used an Arabidopsis microarray-based gene expression analysis tool (Arabidopsis eFP Browser, http://bar.utoronto. ca/efp/cgi-bin/efpWeb.cgi) to analyze the expression profiles of the three AtMDL genes in different tissues, during development and upon different abiotic and biotic stress cues. We found that AtMDL1 and in particular AtMDL2 show fairly constitutive expression in most aerial plant tissues and during most developmental stages, whereas expression in roots is comparatively low. In contrast to this broad expression pattern, AtMDL3 expression seems to be restricted to cotyledons, rosette leaves and sepals (i.e. all types of green leaves; Additional file 2: Figure S2A and Additional file 3: Table S1). Overall, AtMDL1 and AtMDL2 show higher basal transcript levels than AtMDL3, but reveal little alteration in transcript levels upon abiotic or biotic stress cues. By contrast, AtMDL3 exhibits strongly enhanced transcript accumulation in leaves upon cold treatment $\left(12 \mathrm{~h}\right.$ at $\left.4^{\circ} \mathrm{C}\right)$ and a less dramatic increase following osmotic stress, wounding and UV-B treatment (Additional file 2: Figure S2B and Additional file 3: Table S1). AtMDL3 shows also elevated transcript levels in leaves upon exposure to various biotic stresses, including microbial elicitors (e.g. treatment with the peptide flg22) and pathogens (e.g. inoculation with Botrytis cinerea, Pseudomonas syringae, Phytophthora infestans or Golovinomyces orontii; Additional file 2: Figure S2C and Additional file 3: Table S1). Taken together, AtMDL1 and AtMDL2 reveal comparatively constant expression levels in most organs, tissues and conditions, whereas AtMDL3 is characterized by lower basal levels, but elevated transcript accumulation in response to various abiotic and biotic stress cues.

\section{Co-expressed genes and interacting proteins}

Co-expression of genes has recently been recognized as one possible predictor of gene products that cooperate in a given process or biochemical pathway $[29,48,49]$. Accordingly, a number of databases have been established to explore potential gene co-expression. We used the online tool ATTED-II (http://atted.jp/) to identify Arabidopsis genes that are co-expressed with the three
AtMDL genes. For each gene we retrieved a list of the top 300 co-expressed genes and compared these for potential communalities. We noted that 67 of the 300 genes co-expressed with AtMDL1 and AtMDL2 are identical (Additional file 4: Table S2), suggesting that the two genes are largely expressed in the same conditions, together with a shared set of genes. Among these common genes is a conspicuously high number of genes coding for cytosolic or plastid-specific ribosomal proteins (23 in total). We subjected the co-expressed genes to PageMan-based overrepresentation analysis (http:// mapman.mpimp-golm.mpg.de/general/ora/ora.shtml). This indeed revealed statistically highly significant $(\mathrm{p}<$ 1e-10) overrepresentation of genes encoding cytoplasmic (AtMDL2) and plastid-specific (AtMDL1) ribosomal proteins (Additional file 4: Table S2; sheet "overrepresented BINs"). Interestingly, human ribosomal protein RPS19 has been identified to interact with $H s$ MIF [50], suggesting that association with ribosomes and/or ribosomal proteins could be a general feature of MIF-like proteins. Notably, none of the genes that were found to be coexpressed with AtMDL1 or AtMDL2 is co-expressed with $A t M D L 3$ or vice versa, indicating that AtMDL1 and AtMDL2 on the one hand and AtMDL3 on the other hand may function in fundamentally different biological processes. We noted the occurrence of several genes firmly implicated in plant defence among the top 300 genes co-expressed with AtMDL3, including PEN2 [51], PEN3 [52], PAD4/SAG101 [53], SID2 [54], EFR [55], SOBIR1 [56], different WRKY genes [57], as well as MLO2 and MLO12 [58]. However, AtMDL3 is not an integral part of the previously described defence regulon [48], although the genes of the defence regulon and the set of AtMDL3-co-expressed genes partially overlap (e.g. MLO2, PEN2 and PEN3). In further support of a putative function in plant immunity, PageMan analysis indicated statistically highly significant $(\mathrm{p}<1 \mathrm{e}-10)$ overrepresentation of receptor kinases among the genes co-expressed with AtMDL3 (Additional file 4: Table S2; sheet "overrepresented BINs"). In sum, the results of the coexpression analysis are consistent with the expression profiles of the three $A t M D L$ genes, which indicated mostly constitutive expression of AtMDL1 and AtMDL2 and stress-inducible expression of AtMDL3 (see above).

We next interrogated the Plant Interactome Database (http://interactome.dfci.harvard.edu-/A_thaliana/) for potentially known physical interactors of the three $A t \mathrm{MDL}$ proteins. This revealed one identified interactor each (on the basis of large-scale yeast two-hybrid screens; [59]) for AtMDL1 and AtMDL3. The protein interacting with AtMDL1 is At2g47590 (photolyase/Blue Light Receptor 2/ BLR2), a protein implicated in DNA repair, while AtMDL3 interacted with At5g64160, a protein of unknown function. The biological relevance of these interaction partners 
identified by yeast-based methods remains, however, to be shown.

\section{MDL proteins in other plant species}

To explore to which extent $M D L$ genes are also present in other plant species, we performed BLAST searches with a focus on fully sequenced and annotated plant genomes at the Plant Genome Database (PlantGDB, http:// www.plantgdb.org/prj/GenomeBrowser/). We included species that represent different levels of plant evolution, i.e. green algae (Chlamydomonas reinhardtii and Volvox carteri), a bryophyte (moss; Physcomitrella patens), a lycophyte (fern relative; Selaginella moellendorffii), a gymnosperm (Picea sitchensis; Sitka spruce) and various angiosperms (monocotyledonous and dicotyledonous plant species). In some cases we found MDL proteins with otherwise identical amino acid sequences but differing C-termini in the database. These likely comprise annotated splice variants of the same gene locus, as reported above for AtMDL1 and AtMDL2 (see above). For consistency, in these cases we only included the variants that seemingly resemble the AtMDL versions, although we cannot rule out that some of the putative splice variants might be biologically meaningful. Similarly, we removed variants that are nearly identical and possibly just the result of sequencing errors or natural genetic variation within a species.
We discovered that each species harbors genes encoding MDL proteins, with typically multiple copies (paralogs) present per species, except the non-vascular plants (green algae and the moss), which encode a single MDF protein each (Table 2). We also noted a trend towards a higher number of MDL paralogs in dicotyledonous than in monocotyledonous plant species, with an average of 3.2 paralogs per dicotyledonous species and no species having less than three paralogs, compared to two paralogs per monocotyledonous species.

Using CLUSTALW2 we generated a multiple sequence alignment of the curated set of plant MDL proteins identified in the context of our BLAST searches (Additional file 5: Figure S3). This analysis revealed seven invariant amino acids (corresponding to $\mathrm{M}^{1}, \mathrm{P}^{2}, \mathrm{~N}^{9}, \mathrm{P}^{35}, \mathrm{~A}^{59}, \mathrm{G}^{67}$ and $\mathrm{R}^{95}$ in the AtMDLs) plus a number of highly conserved residues. Each of the invariant residues is also present in $H s \mathrm{MIF}$ and $H s \mathrm{DDT}$ (Figure 1), suggesting that these are crucial amino acids for the structure and/or function of MDL proteins. Overall, there is good conservation of amino acid sequences along the entire plant proteins, except at the very $\mathrm{C}$-terminus. As expected, the angiosperm MDL sequences showed the highest level of conservation, while the sequences of the more distantly related gymnosperms, the lycophyte $S$. moellendorffii and the nonvascular plants (green algae and moss) were more variable.

Table 2 Plant MDL proteins

\begin{tabular}{|c|c|c|c|c|}
\hline & Scientific name & Common name & $\begin{array}{l}\text { Number of MDL } \\
\text { proteins }\end{array}$ & $\begin{array}{l}\text { Number of MDL proteins with PTS1- } \\
\text { like motif }\end{array}$ \\
\hline Green algae & $\begin{array}{l}\text { Chlamydomonas } \\
\text { reinhardtii }\end{array}$ & n.a. ${ }^{a}$ & 1 & 0 \\
\hline Green algae & Volvox carteri & n.a. & 1 & 0 \\
\hline Moss & Physcomitrella patens & $\begin{array}{l}\text { Spreading-leaved earth } \\
\text { moss }\end{array}$ & 1 & 0 \\
\hline Lycophyte (fern relative) & $\begin{array}{l}\text { Selaginella } \\
\text { moellendorffii }\end{array}$ & Spike moss & 4 & 0 \\
\hline Gymnosperm & Picea sitchensis & Sitka spruce & 2 & 0 \\
\hline \multirow[t]{5}{*}{$\begin{array}{l}\text { Monocotyledonous } \\
\text { angiosperms }\end{array}$} & $\begin{array}{l}\text { Brachypodium } \\
\text { distachion }\end{array}$ & Purple false brome & 2 & 0 \\
\hline & Hordeum vulgare & Barley & 2 & 0 \\
\hline & Oryza sativa & Rice & 2 & 0 \\
\hline & Sorghum bicolor & Sorghum & 2 & 0 \\
\hline & Zea mays & Maize & 2 & 0 \\
\hline \multirow{6}{*}{$\begin{array}{l}\text { Dicotyledonous } \\
\text { angiosperms }\end{array}$} & Arabidopsis thaliana & Thale cress & 3 & 1 \\
\hline & Glycine max & Soybean & 4 & 1 \\
\hline & Lotus japonicus & Birdsfoot trefoil & 3 & 1 \\
\hline & Prunus persica & Peach & 3 & 1 \\
\hline & Solanum lycopersicon & Tomato & 3 & 1 \\
\hline & Vitis vinifera & Grapevine & 3 & 1 \\
\hline
\end{tabular}

a.a., not applicable. 
The multiple sequence alignment revealed that most plant MDL proteins harbor either one or no cysteine residue. Exceptions are the algal MDL sequences of $C$. reinhardtii and $V$. carteri, which include two cysteine residues, and one of the $S$. moellendorfii sequences with three cysteines (Additional file 6: Figure S4). If present, the cysteine residues are in positions different from those seen in HsMIF. Thus, the vast majority of plant MDL proteins lack the capacity to form an intramolecular disulphide bridge.

We recognized the occurrence of potential variants of the C-terminal PTS1 peroxisome localization signal in some of the sequences. Closer inspection of these amino acid sequences with PTS1 predictor (http://mendel.imp.ac.at/ mendeljsp/sat/pts1/PTS1predictor.jsp) indeed corroborated the presence of likely PTS1 sequences in most of these proteins, while prediction results were ambiguous for two of the analyzed polypeptides (G. $\max$ ACU19241 and $L$. japonicus AFK35159). These findings suggest that not only AtMDL3, but also MDL proteins of other plant species localize to peroxisomes. We noted that each dicotyledonous plant seems to encode one putatively peroxisome-localized MDL paralog, while these were apparently absent from monocotyledonous plants and outside the angiosperm lineage (Additional file 5: Figure S3). This notion was further substantiated by dedicated BLAST searches using AtMDL3 as a query sequence, which revealed that PTS1-carrying MDL sequences appear to be restricted to dicotyledonous plant species. A basic isoelectric point as recognized for AtMDL3 (Table 1) seems to be a common feature of these MDL proteins, as they all exhibit calculated pI values of 8.8-9.2.

Unlike the predicted PTS1 signal in AtMDL3, which could be also recognized in several paralogs from other plant species, the predicted NES in AtMDL1 could not be confirmed in the AtMDL1 relatives. Although all amino acid sequences from this clade show a tendency towards an NES in the respective region (corresponding to amino acid 85-91 in AtMDL1), the threshold for a positive prediction is only passed in the case of AtMDL1 (owing to the presence of an isoleucine in position 87). The relevance of these in silico analyses remains to be tested experimentally.

\section{Phylogenetic analysis of plant MDL proteins}

We extended our study by performing phylogenetic analysis of the subset of plant MDL protein sequences applying two widely used computational phylogenetic methods (Neighbor-Joining and Maximum Likelihood). Results of these analyses consistently revealed that AtMDL1 is a member of a clade that comprises sequences from both mono- and dicotyledonous plant species (Figure 3; see also Additional file 7: File S2 for
Newick notation of the phylogenetic trees). By contrast, results differ for AtMDL2. While the distance matrixbased Neighbor-Joining method indicates that the AtMDL2 clade also harbors sequences from both monoand dicotyledonous plant species (Figure 3A), the probability-based Maximum Likelihood tree separates MDL members from these two lineages (Figure 3B). In both phylogenetic trees the two sequences of the gymnosperm plant $P$. sitchensis each group as sister branches of these major clades, suggesting that the respective dichotomous split occurred before the gymnosperm-angiosperm separation ca. 285 million years ago [60]. In the Neighbor-Joining tree, the moss $(P$. patens) MDL sequence groups together with the AtMDL1 clade and one of the four lycophyte (S. moellendorffii) sequences, suggesting that this branch reflects the most ancestral MDLs of land plants. This issue remains, however, unresolved in the Maximum Likelihood tree.

AtMDL3 is a member of a discrete clade, containing exclusively sequences of dicotyledonous MDL proteins with a predicted C-terminal peroxisome localization signal. This clade is a sister clade of the AtMDL2-containing clade, suggesting that the AtMDL3 lineage originates from a gene duplication event, involving a common progenitor of AtMDL2 and AtMDL3, after the divergence of monocotyledonous and dicotyledonous plant species ca. 145 million years ago [61]. As expected, human $H s$ MIF and $H s \mathrm{DDT}$ cluster as out-groups in both trees and show closest relationship to MDL sequences from primitive plants such as green algae and three out of the four lycophyte MDLs.

Based on the phylogenetic separation we established dedicated clade-specific sequence alignments of AtMDL1-, AtMDL2- and AtMDL3-like angiosperm MDL proteins (Additional file 8: Figure S5). This revealed that AtMDL2like sequences show the highest degree of sequence conservation, with 72 invariant amino acids within this clade, as compared to 56 and 46 invariant amino acids in AtMDL1- and AtMDL3-like sequences, respectively.

\section{Discussion}

Our in silico analysis revealed that plant MDL proteins resemble $H s \mathrm{MIF}$ and $H s \mathrm{DDT}$ with regard to primary amino acid sequence and predicted three-dimensional structure (Figures 1 and 2). Together with the presence of this type of protein in other kingdoms of life (e.g. in protists [41] and eubacteria [62]), this finding suggests a common evolutionarily preserved core biochemical function for MDL proteins. Human MIF is a multifunctional polypeptide with at least three biological activities. First, it serves a role as an extracellular cytokine/chemokine that binds to the HLA class II histocompatibility antigen gamma chain (also known as the cell surface receptor CD74) or to the classical CXC chemokine receptors 


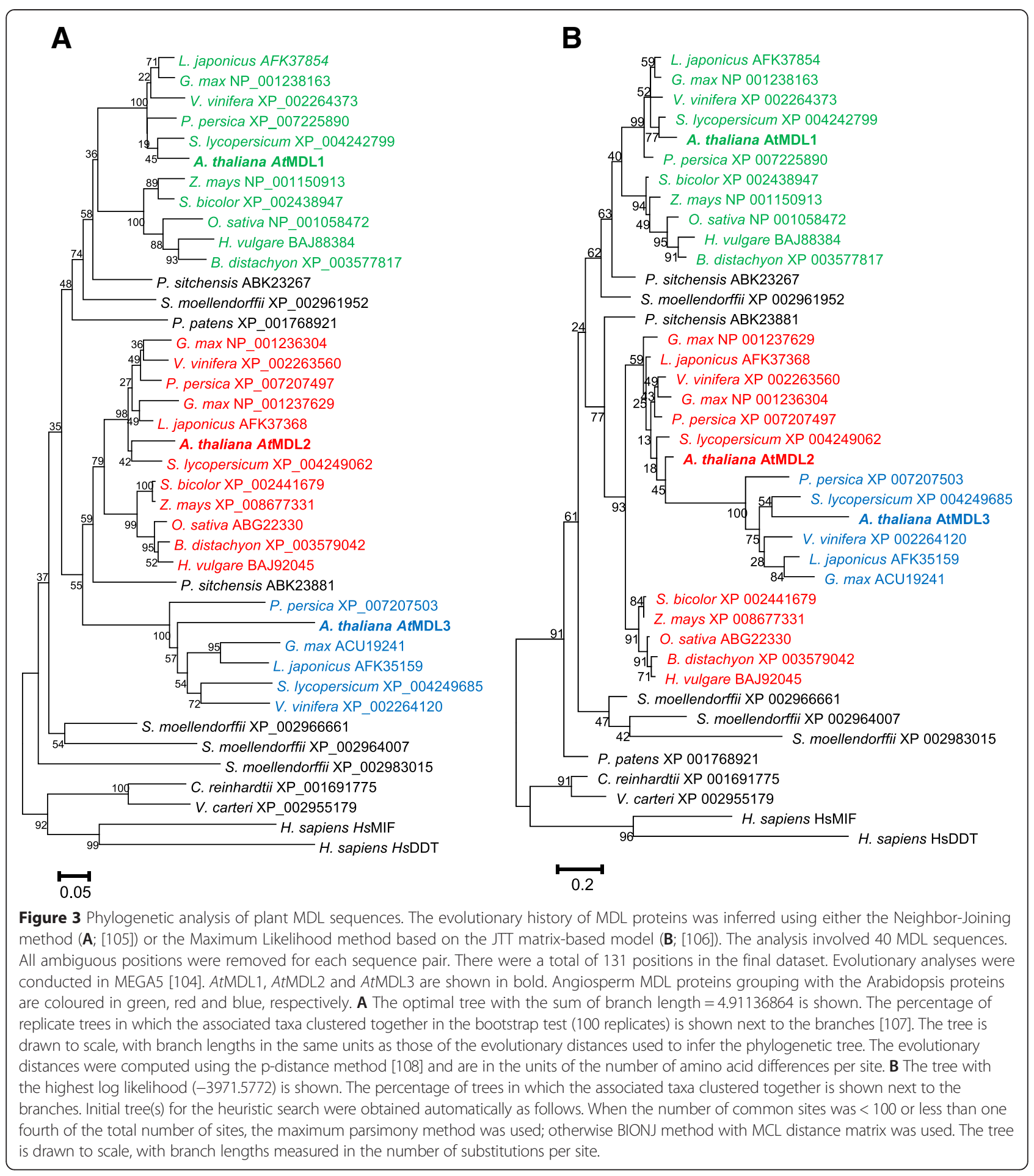

CXCR2 and CXCR4, thereby modulating innate immune responses $[14,19,20]$. The significance of a more broad relationship of $H s$ MIF to classical chemokine receptors was most recently corroborated by the discovery of $H s \mathrm{MIF} / \mathrm{CXCR7}$ interactions on platelets [63]. Of note, this suggests that CXCR7 shares with CXCR4 not only the cognate ligand CXCL12, but also interacts with $H s \mathrm{MIF}$,
[14]. In addition, $H s$ MIF has two physically separated catalytic sites that confer tautomerase as well as oxidoreductase activity, although the authentic in vivo substrates of these enzymatic activities remain unknown $[34,64]$. The contribution of these catalytic activities to $H s$ MIF's chemokine function is controversially discussed and has not been fully resolved $[10,13,65,66]$. 
Plant MDL proteins seem to lack at least two of these three roles, namely chemokine function and oxidoreductase activity. Unlike vertebrates, plants do not have professional mobile immune cells such as macrophages. Instead each plant cell largely relies on its own capacity to combat microbial intruders (cell-autonomous immunity; $[67,68])$. Consistent with this type of innate immunity, plants do not possess components such as chemokines that could attract migrating immune cells to biotic stress sites. Plant MDL proteins are therefore unlikely to be secreted as signaling molecules to the extracellular space. This notion is supported by the absence of a recognizable $\mathrm{N}$-terminal secretion signal and the lack of the so-called pseudo-ELR motif. Lack of a canonical N-terminal secretion signal in $H s \mathrm{MIF}$ in conjunction with experimental findings has led to the view that human MIF and DDT are secreted via unconventional secretion pathways $[42,43,69]$. It nevertheless remains at least a formal possibility that also plant MDL proteins exert an extracellular function. In favor of this latter notion would be the finding that AtMDL3 and related plant MDLs exhibit a fairly basic pI value above 8 (Table 1). HsMIF has a pI value of 7.73 and it is believed that this feature contributes to endothelial deposition of extracellular MIF, similar to the well-known arrest chemokines [20]. The some 50 classical chemokines generally exhibit basic $\mathrm{pI}$ values in the range between 7.5 and 8.8 and it is this biophysical property that guarantees their endothelial immobilization following secretion on the extensively acidic proteoglycan ("glycocalix") layer of the endothelium. Once immobilized, chemokines form a haptotactic gradient enabling them to mediate leukocyte arrest and transmigration during homeostatic homing processes or in inflammatory leukocyte extravasation $[70,71]$. In fact, acidic proteoglycans have been proposed to function as co-receptors in leukocyte arrest $[72,73]$. Biochemical evidence for direct MIF-proteoglycan interaction is still missing, but both human and mouse MIF are known to be deposited on the endothelial surface following secretion from endothelial cells and/or leukocytes and thrombocytes, promoting leukocyte activation and arrest [20,74]. Moreover, the surface form of CD74/invariant chain, a receptor for human and mouse MIF, is chondroitin sulfate-modified, a posttranslational prerequisite necessary for high affinity interaction with MIF [75].

Two closely linked cysteine residues form the central part of the TPOR site that is required for oxidoreductase activity in $H s$ MIF (Figure. 1; [22,34]). These paired cysteines are thought to serve a thioredoxin-like role during redox reactions catalyzed by $H s$ MIF. To also exert such an enzymatic activity the presence of paired cysteine residues would be expected in plant MDL proteins. However, most plant MDLs either have none or only one cysteine residue, with only few plant MDLs harboring two or more cysteines (Additional file 6: Figure S4). Exceptions are the algal MIFs encoded by the C. reinhardtii and $V$. carteri genomes, which each possess two similarly spaced cysteines in the same relative position (Additional file 6: Figure S4). Based on these amino acid features it is conceivable that at least the majority of plant MDLs do not possess oxidoreductase activity. This is in agreement with MIF-like proteins from several other non-vertebrate species, which were also suggested to lack this catalytic capacity $[41,62,76]$. However, it seems that plant MDLs have the principal potential to share tautomerase activity with their counterparts from other kingdoms of life (animals and protists). Evidence for this assumption is conservation of an N-terminal proline residue that is known to form the catalytic site for tautomerase activity (Figure 1). Since to our knowledge this sequence feature is present in all MIF proteins known so far, tautomerase activity could be the ancestral function of MDLs. Although no in vivo tautomerase substrate has been identified, the presumed conservation of tautomerase activity may hint at a chemical or chemical class whose presence is conserved across a broad range of taxa. D-dopachrome has been recognized as an artificial substrate of $H s$ MIF and other MDLs [42]. This compound is a cyclization product of L-3,4-dihydroxyphenylalanine (also known as L-DOPA) and an intermediate in the biosynthesis of melanin-type pigments. L-DOPA is best known for its role as a precursor molecule of various human neurotransmitters, but it also exists in plants where it seems to serve a role as precursor of different secondary plant metabolites [77]. Although plants lack conventional melanins, they are able to synthesize catechol melanin, which is also chemically related to L-DOPA [78]. It remains to be seen whether the natural substrate of MDL's tautomerase activity is indeed related to L-DOPA.

We observed a tendency towards an increased copy number of $M D L$ genes with increasing organismal complexity within the plant lineage. While evolutionarily older non-vascular plants such as algae and mosses contain only one $M D L$ copy, evolutionarily younger vascular plants contain two or more paralogs (Table 2). A further increase in complexity of the $M D L$ gene family can be seen within the angiosperm clade: While monocotyledonous plant species typically have two $M D L$ copies, dicotyledonous species encode three or more MDL paralogs (Table 2). The additional copy in dicotyledonous plant species represents a type of MDL with unique gene and protein features. This MDL type, in Arabidopsis represented by AtMDL3, has a C-terminal peroxisome targeting sequence (PTS1) and a different overall amino acid composition, as reflected by a markedly higher isoelectric point and a separate position within the phylogenetic tree (Tables 1 and 2, Figure 3). 
Moreover, the respective gene has at least in Arabidopsis a different genomic organization (lacking one intron; Figure 1), is the only Arabidopsis $M D L$ that shows stimulus-dependent expression, and is co-expressed with a unique gene set (Additional file 4: Table S2). Together, these features point to neofunctionalization of this plant MDL paralog in dicotyledonous plants.

The Arabidopsis product of this MDL paralog (AtMDL3) is predicted (by presence of the C-terminal PTS1 sequence; Table 1) and has been experimentally shown (by proteomic and cell biological analysis; $[46,47]$ ) to localize to peroxisomes. Plant peroxisomes are involved in numerous biological processes, including primary and secondary metabolism, development, as well as responses to biotic and abiotic stress cues. Similar to peroxisomes in mammalian cells, they are best known for their role in fatty acid oxidation ( $\beta$-oxidation). In plants, they are also involved in the biosynthesis of the phytohormones indole acetic acid (IAA, an auxin) and jasmonic acid and they contribute to the process of photorespiration [79]. Transcript accumulation of AtMDL3 is responsive to various abiotic and biotic stress stimuli, indicating that the respective protein may play a role in these conditions. Indeed, peroxisomes have been found to be responsive to abiotic stress $[80,81]$ and biotic stress $[82,83]$. With regard to the latter, PEN2, an atypical myrosinase presumably involved in the biosynthesis of toxic glucosinolate metabolites, has been found to be associated with peroxisomes $[51,84]$. Notably, PEN2, which is also part of an antifungal defence regulon [48], is in the list of genes co-expressed with AtMDL3 (Additional file 4: Table S2). Accordingly, AtMDL3 may have a role in pathogen defence. If this was true, and if this was a general feature of angiosperm AtMDL3-like proteins, then we may face the situation of convergent evolution in higher plants and mammals/vertebrates, characterized by neofunctionalization of MIFs/ MDLs towards a role in innate immunity.

The remaining two types of plant MDLs (represented by $A t M D L 1$ and $A t M D L 2$ ) show constitutive expression and are both co-expressed with an overlapping set of genes. This essentially resembles the expression profile of $H s M I F$, which was also described to be constitutively expressed in immune cells and stress-responsive tissues of the endocrine system [10]. Among the genes coexpressed with AtMDL1 and AtMDL2 are a remarkably high number of genes encoding ribosomal proteins. Notably, HsMIF has been reported to interact with ribosomal protein S19 (RPS19), thereby attenuating its proinflammatory function [50]. As RPS19 can be released in inflammatory lesions by apoptotic cells, it has been suggested to act as an extracellular negative regulator of MIF function in this context [50]. Co-expression with a greater number of genes coding for ribosomal proteins suggests that at least AtMDL1 and AtMDL2 may act together with ribosomes, e.g. during protein biosynthesis. The similar expression pattern and an overlapping set of co-expressed genes further indicate that the two genes might be functionally redundant in Arabidopsis. Owing to a lack of dedicated targeting sequences, one might expect that the AtMDL1 and AtMDL2 proteins localize to the cytoplasm and/or the nucleus (because of passive diffusion). The functional relevance of a putative NES detected in AtMDL1 awaits experimental verification.

Only two proteins have so far been identified as interaction partners of Arabidopsis MDLs. According to largescale yeast two-hybrid data AtMDL1 interacts with At2g47590 (photolyase/Blue Light Receptor 2/BLR2) and AtMDL3 interacts with At5g64160 (a protein implicated in DNA repair; [59]). None of these interactions has been validated in planta so far. In contrast to the AtMDL proteins, a substantial number of interacting proteins have been identified for $H s$ MIF. These comprise PAG [85], JAB/CSN5 [86], BNIPL [87], HPO [88] and RPS19 ([50]; see also above). Since currently no common theme or interrelation of these interaction partners can be recognized, one may speculate that $H s$ MIF functions through a number interactions with a diverse set of proteins.

To further unravel the function of plant MDLs, genetic, molecular and cell biological analyses will be necessary. T-DNA insertion lines for all three AtMDL genes can be found in public repositories (http://signal.salk. edu/cgi-bin/tdnaexpress) and could be used to identify potential phenotypes of AtMDL knock-out plants. Further protein-protein interaction screens are required to uncover additional interaction partners of the Arabidopsis MDL proteins. Moreover, in planta assays will be necessary to validate these yeast-based interactors. Subcellular localization studies using fluorophore-tagged proteins will shed light on the cellular site of action of the Arabidopsis MDLs. Analysis of plant MDLs may help to finally get a hold of the long-sought after natural substrates of the MIF/DDT tautomerase activities.

\section{Conclusions}

We performed an in depth in silico analysis of plant MDL proteins. This unraveled that MIF/DDT-like proteins are present in all plant taxa, with an increasing number of paralogs in higher plant species. Plant MDLs share extensive sequence similarity (Figure 1) and predicted 3D structure (Figure 2) with their non-plant counterparts. They are predicted to lack oxidoreductase activity but possibly retained tautomerase activity as catalytic function. Phylogenetic analysis (Figure 3) allowed reconstruction and time estimates of the likely phylogeny of the MDL protein family in the course of plant evolution. The genome of the dicotyledonous model plant species Arabidopsis thaliana harbors three 
$M D L$ genes, of which two are constitutively expressed and share an overlapping set of co-expressed genes, many of which code for ribosomal proteins. The third gene exhibits stress-inducible transcript accumulation and is co-expressed with a number of genes implicated in plant immunity. This gene encodes an MDL variant (AtMDL3) with a C-terminal peroxisomal targeting signal (PTS1). The (co-) expression pattern and subcellular localization of AtMDL3 suggest convergent evolution in higher plants and vertebrates towards MDL variants with a novel role in innate immunity. Precedence for such a scenario is provided by the convergent neofunctionalization of xanthine dehydrogenase towards aldehyde oxidase after recurring gene duplication events [89]. Functional analyses will be required to validate this hypothetical convergent neofunctionalization of MDL proteins in two separate kingdoms of life.

The comprehensive characterization of plant MDL proteins as undertaken in this study should aid in understanding the broader biological role of these proteins across kingdoms and should form the basis for future experimental work on these proteins. The catalytic activities of MIF have been extensively studied by numerous laboratories across the globe for more than 15 years. However, most of these studies have focused on the pure in vitro activity. Moreover, the physiologically relevant substrates have remained elusive and the functional role of these activities in MIF's physiologic and pathophysiologic activities in humans and rodents is still unclear and under debate. Studying the catalytic activities of MDL proteins in plants and identifying novel MDL interaction partners could thus aid in uncovering the long soughtafter natural substrates.

A broad body of evidence now clearly suggests that $H s$ MIF is an evolutionarily conserved protein that has both intra- and extracellular functions. In the human and mouse studies, the extracellular activities of MIF have been primarily addressed, with mostly only indirect evidence obtained about its intracellular effects. We therefore hypothesize that clarifying MIF's role in a remote organism/kingdom such as plants in which a circulation/extracellular space-based mobile immune and defense system is missing, could provide valuable novel information about MIF's intracellular effects, assuming that these are highly conserved and likely appeared first in the evolution of MIF proteins.

\section{Methods}

\section{Sequences and BLAST analysis}

The amino acid sequences used in this study were retrieved from NCBI/GenBank and can be found in Additional file 9: File S1. BLAST searches were performed against the NCBI (http://blast.st-va.ncbi.nlm.nih.gov/Blast.cgi), TAIR (http:// www.arabidopsis.org/Blast/index.jsp) and Plant Genome
Database (PlantGDB; http://www.plantgdb.org/prj/Genome Browser/), respectively. AtMDL gene models were retrieved from TAIR (http://www.arabidopsis.org/).

\section{Multiple sequence alignments}

Multiple amino acid sequence alignments were established with CLUSTALW2, a general purpose DNA/ protein multiple sequence alignment program (http:// www.ebi.ac.uk/Tools/msa/clustalw2/; [90]) using standard parameters. Shading of alignments for conserved amino acids was performed with the help of BoxShade 3.21 (http://www.ch.embnet.org/software/BOX_form.html).

\section{Structure prediction and visualization}

Structure prediction was carried out with the Phyre ${ }^{2}$ fold recognition server (http://www.sbg.bio.ic.ac.uk/ phyre2/html/page.cgi?id=index; [91]) and the QUARK server (http://zhanglab.ccmb.med.umich.edu/QUARK/; [92]) using standard parameters. Phyre ${ }^{2}$ uses homology modeling based on the alignment of hidden Markov models. It also incorporates $a b$ initio folding simulation to model regions of proteins with no detectable homology to known structures. QUARK models are built from small fragments (1-20 residues long) by replicaexchange Monte Carlo simulation under the guide of an atomic-level knowledge-based force field. Calculated 3D structures were visualized with PyMOL (http://www. pymol.org/; The PyMOL Molecular Graphics System, Version 1.7.4 Schrödinger, LLC), either as a cartoon showing the secondary structures ( $\alpha$-helices and $\beta$-sheets) or as a space-filling model revealing the electrostatic surface potential. The latter is calculated by averaging charges over a small region of space using a quasi-Coulombic-shaped convolution function (charge-smoothed potential).

\section{Online tools}

Protein parameters such as molecular mass and isoelectric point were calculated with ExPASy bioinformatics resource portal (http://www.expasy.org/). The presence of signal peptides and transmembrane domains was explored with SignalP 4.1 (neural network-based prediction of the presence and location of signal peptide cleavage sites in amino acid sequences from different organisms; http:// www.cbs.dtu.dk/services/SignalP/; [93]) and TMHMM 2.0 (hidden Markov model-based prediction of the presence and location of transmembrane helices in proteins; http:// www.cbs.dtu.dk/services/TMHMM/; [94]), respectively. Subcellular protein localization was analyzed via Mitoprot (prediction of mitochondrial targeting sequences; http:// ihg.gsf.de/ihg/mitoprot.html; [95]), ChloroP 1.1 (neural network-based prediction of chloroplast transit peptides; http://www.cbs.dtu.dk/services/ChloroP/; [96]), NLStradamus (hidden Markov model-based prediction of nulear localization signals; http://www.moseslab.csb.utoronto.ca/ 
NLStradamus/; [97]) NetNES 1.1 (a combined neural network and hidden Markov model-based prediction of nuclear export sequences; http://www.cbs.dtu.dk/services/ NetNES/; [98]) and PTS1 predictor (prediction of peroxisomal targeting sequence 1; http://mendel.imp.ac.at/mend eljsp/sat/pts1/PTS1predictor.jsp; [99]).

Searches for protein domains were performed with InterProScan 5, which scans for matches against the InterPro collection of protein signature databases (http://www. ebi.ac.uk/Tools/pfa/iprscan5/; [100]). Arabidopsis gene expression data were retrieved via the Arabidopsis eFP browser, a pictographic exploration tool for microarray data (http://bar.utoronto.ca/efp/cgi-bin/efpWeb.cgi; [101]). Coexpression analysis was performed using ATTED-II, a database that uses the weighted Pearson's correlation coefficient to compare microarray-based gene expression profiles to reveal co-expression (http://atted.jp/; [102]). Arabidopsis protein-protein interaction data were obtained from the Plant Interactome Database (http://inter actome.dfci.harvard.edu/A_thaliana/), which represents a repository of large-scale yeast two-hybrid results. Overrepresentation of co-expressed genes was analyzed with the ORA feature of the PageMan tool, which allows effective comparative analysis of multiple microarray experiments (http://mapman.mpimp-golm.mpg.de/general/ora/ora.shtml; [103]) using the TAIR8 Arabidopsis protein set as a control group.

\section{Phylogenetic analysis}

The evolutionary history of plant MDL proteins was reconstructed with Neighbor-Joining and Maximum Likelihood analysis on the basis of CLUSTALW-aligned MIF/MDL sequences using MEGA5 software [104]. For the Neighbor-Joining tree, the p-distance amino acid substitution model was applied, using uniform rates among sites and pairwise deletion. For the Maximum Likelihood tree, the Jones-Thornton-Taylor (JTT) model was applied, using uniform rates and all sites. The Nearest NeighborhoodInterchange (NNI) method was employed for inference of the Maximum Likelihood tree. Both procedures (NeighborJoining and Maximum Likelihood) use the bootstrap method with 100 replicates for testing the phylogeny.

\section{Additional files}

Additional file 1: Figure S1. Ab initio prediction of HsMIF/AtMDL 3D structures with QUARK. Amino acid sequences of the AtMDLs were subjected to analysis via the QUARK 3D structure prediction server (http://zhanglab.ccmb.med.umich.edu/QUARK/) and rendered with PyMOL (http://www.pymol.org/). The predicted 3D structures (ribbon models) of AtMDL1, AtMDL2 and AtMDL3 are visualized in comparison to the predicted structure of HSMIF.

Additional file 2: Figure S2. AtMDL expression profiles. Gene expression data for AtMDL1, AtMDL2 and AtMDL3 are shown according to the Arabidopsis eFP browser and represent data sets from development (A), abiotic (B) and biotic (C) stress.

Additional file 3: Table S1. AtMDL expression profiles. Gene expression data for AtMDL1, AtMDL2 and AtMDL3 are shown according to the Arabidopsis eFP browser (http://bar.utoronto.ca/efp/cgi-bin/efpWeb.cgi) and represent data sets from development, abiotic and biotic stress.

Additional file 4: Table S2. AtMDL co-expression. Sheet "AtMDL1": Top 300 genes co-expressed with AtMDL1 according to ATTED-II ((http:// atted.jp/). Highlighted in red are those genes that overlap with genes that are co-expressed with AtMDL2. Sheet "AtMDL2": Top 300 genes co-expressed with AtMDL2 according to ATTED-II (http://atted.jp/). Highlighted in red are those genes that overlap with genes that are co-expressed with AtMDL1.Sheet"AtMDL3": Top 300 genes co-expressed with AtMDL3 according to ATTED-II (http://atted.jp/) Sheet "overrepresented BINs": Results of overrepresentation analysis using PageMan (http://mapman .mpimp-golm.mpg.de/general/ora/ora.shtml). Shown are the results of BINs that exhibit a p-value of <1e-10.

Additional file 5: Figure S3. Multiple sequence alignment of plant MDL proteins. Plant MDL amino acid sequences were aligned with ClustalW2 (http://www.ebi.ac.uk/Tools/msa/clustalw2/) using standard parameters. Subsequently, the alignment was shaded with BoxShade 3.21 (http://www.ch.embnet.org/software/BOX_form.html) using standard settings. A black shade indicates identical amino acids; a grey shade denotes similar amino acids. In the consensus line, asterisks specify invariant amino acids and dots conserved amino acids with similar biophysical properties at a given position.

Additional file 6: Figure S4. Multiple sequence alignment of plant MDL proteins highlighting the distribution of cysteine residues. Plant MDL amino acid sequences were aligned with ClustalW2 (http:// www.ebi.ac.uk/Tools/msa/clustalw2/) using standard parameters. Cysteine residues are highlighted by yellow background.

Additional file 7: File S2. Newick annotation of phylogenetic trees shown in Figure 3.

Additional file 8: Figure S5. Multiple sequence alignments of AtMDL1-, AtMDL2- and AtMDL3-like proteins. Plant MDL amino acid sequences were aligned with ClustalW2 (http://www.ebi.ac.uk/Tools/msa/clustalw2/) using standard parameters. Subsequently, the alignment was shaded with BoxShade 3.21 (http://www.ch.embnet.org/software/BOX_form.html) using standard settings. A black shade indicates identical amino acids; a grey shade denotes similar amino acids. In the consensus line, asterisks specify invariant amino acids and dots conserved amino acids with similar biophysical properties at a given position.

Additional file 9: File S1. MIF/MDL protein sequences used in this study.

\section{Abbreviations}

DDT: D-dopachrome tautomerase; MDL: MIF/DDT-like gene/protein; MIF: Macrophage migration inhibitory factor; NES: Nuclear export signal; PTS1: Peroxisomal targeting sequence 1; TPOR: Thiol-protein oxidoreductase.

\section{Competing interests}

The authors declare that they have no competing interests.

\section{Authors' contributions}

JB and RP designed the study, RP created the Figures, KB and RP analyzed the data, and JB and RP wrote and edited the manuscript. All authors read and approved the final manuscript.

\section{Acknowledgements}

This work was supported by DFG grants SFB1123/A03 and SFB-TRR57/P07 as well as IZKF Aachen grant O2-2 to J.B.

\section{Author details}

${ }^{1}$ RWTH Aachen University, Institute of Biology I, Unit of Plant Molecular Cell Biology, Worringerweg 1, 52074 Aachen, Germany. ${ }^{2}$ RWTH Aachen University, Institute of Biochemistry and Molecular Cell Biology, Pauwelsstrasse 30, 52074 Aachen, Germany. 
Received: 17 November 2014 Accepted: 19 March 2015 Published online: 14 April 2015

\section{References}

1. Mantovani A. The chemokine system: redundancy for robust outputs. Immunol Today. 1999;20:254-7

2. Zlotnik A, Yoshie O. Chemokines: a new classification system and their role in immunity. Immunity. 2000;12:121-7.

3. Baggiolini M. Chemokines in pathology and medicine. J Intern Med. 2001;250:91-104.

4. Moser B, Loetscher P. Lymphocyte traffic control by chemokines. Nat Immunol. 2001;2:123-8.

5. Rot A, Von A, Ulrich $\mathrm{H}$. Chemokines in innate and adaptive host defense: basic chemokinese grammar for immune cells. Annu Rev Immunol. 2004;22:891-928.

6. Thelen M, Stein JV. How chemokines invite leukocytes to dance. Nat Immunol. 2008;9:953-9.

7. Charo IF, Ransohoff RM. The many roles of chemokines and chemokine receptors in inflammation. N Engl J Med. 2006;354:610-21.

8. Sallusto F, Baggiolini M. Chemokines and leukocyte traffic. Nat Immunol. 2008;9:949-52

9. Thelen M. Dancing to the tune of chemokines. Nat Immunol. 2001;2:129-34.

10. Calandra T, Roger T. Macrophage migration inhibitory factor: a regulator of innate immunity. Nat Rev Immunol. 2003;3:791-800.

11. Lue H, Kleemann R, Calandra T, Roger T, Bernhagen J. Macrophage migration inhibitory factor (MIF): mechanisms of action and role in disease. Microbes Infect. 2002:4:449-60.

12. Degryse $B$, de Virgilio $M$. The nuclear protein HMGB1, a new kind of chemokine? FEBS Lett. 2003;553:11-7.

13. Noels $\mathrm{H}$, Bernhagen J, Weber C. Macrophage migration inhibitory factor: a noncanonical chemokine important in atherosclerosis. Trends Cardiovasc Med. 2009;19:76-86.

14. Tillmann S, Bernhagen J, Noels $\mathrm{H}$. Arrest functions of the MIF ligand/ receptor axes in atherogenesis. Front Immunol. 2013;4:115.

15. Schober A, Bernhagen J, Weber C. Chemokine-like functions of MIF in atherosclerosis. J Mol Med. 2008;86:761-70.

16. Sun HW, Bernhagen J, Bucala R, Lolis E. Crystal structure at 2.6-Å resolution of human macrophage migration inhibitory factor. Proc Natl Acad Sci U S A. 1996;93:5191-6.

17. Morand EF, Leech M, Bernhagen J. MIF: a new cytokine link between rheumatoid arthritis and atherosclerosis. Nat Rev Drug Discov. 2006;5:399-410.

18. David JR. Delayed hypersensitivity in vitro: its mediation by cell-free substances formed by lymphoid cell-antigen interaction. Proc Natl Acad Sci U S A. 1966:56:72-7.

19. Leng L, Metz CN, Fang Y, Xu J, Donnelly S, Baugh J, et al. MIF signal transduction initiated by binding to CD74. J Exp Med. 2003;197:1467-76.

20. Bernhagen J, Krohn R, Lue H, Gregory JL, Zernecke A, Koenen RR, et al. MIF is a noncognate ligand of CXC chemokine receptors in inflammatory and atherogenic cell recruitment. Nat Med. 2007;13:587-96.

21. Bernhagen J, Calandra T, Mitchell RA, Martin SB, Tracey K, Voelter W, et al. MIF is a pituitary-derived cytokine that potentiates lethal endotoxaemia. Nature. 1993;365:756-9.

22. Thiele M, Bernhagen J. Link between macrophage migration inhibitory factor and cellular redox regulation. Antioxid Redox Signal. 2005;7:1234-48.

23. Qi D, Atsina $K$, Qu L, Hu X, Wu X, Xu B, et al. The vestigial enzyme Ddopachrome tautomerase protects the heart against ischemic injury. J Clin Invest. 2014;124:3540-50.

24. Merk M, Mitchell RA, Endres S, Bucala R. D-dopachrome tautomerase (D-DT or MIF-2): doubling the MIF cytokine family. Cytokine. 2012;59:10-7.

25. Merk M, Zierow S, Leng L, Das R, Du X, Schulte W, et al. The D-dopachrome tautomerase (DDT) gene product is a cytokine and functional homolog of macrophage migration inhibitory factor (MIF). Proc Natl Acad Sci U S A. 2011;108:E577-85.

26. Kobold S, Merk M, Hofer L, Peters P, Bucala R, Endres S. The macrophage migration inhibitory factor (MIF)-homologue D-dopachrome tautomerase is a therapeutic target in a murine melanoma model. Oncotarget. 2014;5:103-7.

27. Koornneef M, Meinke D. The development of Arabidopsis as a model plant. Plant J. 2010;61:909-21.

28. Initiative TAG. Analysis of the genome sequence of the flowering plant Arabidopsis thaliana. Nature. 2000;408:796-815.
29. lida K, Kawaguchi S, Kobayashi N, Yoshida Y, Ishii M, Harada E, et al. ARTADE2DB: improved statistical inferences for Arabidopsis gene functions and structure predictions by dynamic structure-based dynamic expression (DSDE) analyses. Plant Cell Physiol. 2011;52:254-64.

30. Miska KB, Fetterer RH, Lillehoj HS, Jenkins MC, Allen PC, Harper SB. Characterisation of macrophage migration inhibitory factor from Eimeria species infectious to chickens. Mol Biochem Parasitol. 2007;151:173-83.

31. Esumi N, Budarf M, Ciccarelli L, Sellinger B, Kozak CA, Wistow G. Conserved gene structure and genomic linkage for D-dopachrome tautomerase (DDT) and MIF. Mamm Genome. 1998;9:753-7.

32. Bernhagen J, Mitchell RA, Calandra T, Voelter W, Cerami A, Bucala R. Purification, bioactivity, and secondary structure analysis of mouse and human macrophage migration inhibitory factor (MIF). Biochemistry. 1994;33:14144-55.

33. Stamps SL, Fitzgerald MC, Whitman CP. Characterization of the role of the amino-terminal proline in the enzymatic activity catalyzed by macrophage migration inhibitory factor. Biochemistry. 1998;37:10195-202.

34. Kleemann R, Kapurniotu A, Frank RW, Gessner A, Mischke R, Flieger O, et al. Disulfide analysis reveals a role for macrophage migration inhibitory factor (MIF) as thiol-protein oxidoreductase. J Mol Biol. 1998;280:85-102.

35. Murphy PM, Baggiolini M, Charo IF, Hébert CA, Horuk R, Matsushima K, et al. International union of pharmacology. XXII Nomenclature for chemokine receptors. Pharmacol Rev. 2000;52:145-76.

36. Weber C, Kraemer S, Drechsler M, Lue H, Koenen RR, Kapurniotu A, et al. Structural determinants of MIF functions in CXCR2-mediated inflammatory and atherogenic leukocyte recruitment. Proc Natl Acad Sci U S A. 2008:105:16278-83.

37. Wang DY, Kumar S, Hedges SB. Divergence time estimates for the early history of animal phyla and the origin of plants, animals and fungi. Proc Biol Sci. 1999;266:163-71.

38. Blanc G, Barakat A, Guyot R, Cooke R, Delseny M. Extensive duplication and reshuffling in the Arabidopsis genome. Plant Cell. 2000;12:1093-101.

39. Ermolaeva MD, Wu M, Eisen JA, Salzberg SL. The age of the Arabidopsis thaliana genome duplication. Plant Mol Biol. 2003:51:859-66.

40. Sugimoto H, Taniguchi M, Nakagawa A, Tanaka I, Suzuki M, Nishihira J. Crystal structure of human D-dopachrome tautomerase, a homologue of macrophage migration inhibitory factor, at $1.54 \AA$ 年solution. Biochemistry. 1999;38:3268-79.

41. Kamir D, Zierow S, Leng L, Cho Y, Diaz Y, Griffith J, et al. A Leishmania ortholog of macrophage migration inhibitory factor modulates host macrophage responses. J Immunol. 2008;180:8250-61.

42. Merk M, Baugh J, Zierow S, Leng L, Pal U, Lee SJ, et al. The Golgi-associated protein p115 mediates the secretion of macrophage migration inhibitory factor. J Immunol. 2009;182:6896-906.

43. Flieger O, Engling A, Bucala R, Lue H, Nickel W, Bernhagen J. Regulated secretion of macrophage migration inhibitory factor is mediated by a non-classical pathway involving an ABC transporter. FEBS Lett. 2003;551:78-86.

44. Zybailov B, Rutschow H, Friso G, Rudella A, Emanuelsson O, Sun Q, et al. Sorting signals, N-terminal modifications and abundance of the chloroplast proteome. PLoS One. 2008;3:e1994.

45. Olinares, Paul Dominic B, Ponnala L, Van W, Klaas J. Megadalton complexes in the chloroplast stroma of Arabidopsis thaliana characterized by size exclusion chromatography, mass spectrometry, and hierarchical clustering. Mol Cell Proteomics. 2010;9:1594-615.

46. Reumann S, Babujee L, Ma C, Wienkoop S, Siemsen T, Antonicelli GE, et al. Proteome analysis of Arabidopsis leaf peroxisomes reveals novel targeting peptides, metabolic pathways, and defense mechanisms. Plant Cell. 2007:19:3170-93.

47. Li J, Park E, Von A, Albrecht G, Nebenführ A. The FAST technique: a simplified Agrobacterium-based transformation method for transient gene expression analysis in seedlings of Arabidopsis and other plant species. Plant Methods. 2009;5:6

48. Humphry M, Bednarek P, Kemmerling B, Koh S, Stein M, Göbel U, et al. A regulon conserved in monocot and dicot plants defines a functional module in antifungal plant immunity. Proc Natl Acad Sci U S A. 2010;107:21896-901.

49. Horan K, Jang C, Bailey-Serres J, Mittler R, Shelton C, Harper JF, et al. Annotating genes of known and unknown function by large-scale coexpression analysis. Plant Physiol. 2008;147:41-57.

50. Filip A, Klug J, Cayli S, Fröhlich S, Henke T, Lacher P, et al. Ribosomal protein S19 interacts with macrophage migration inhibitory factor and attenuates its pro-inflammatory function. J Biol Chem. 2009;284:7977-85. 
51. Lipka V, Dittgen J, Bednarek P, Bhat R, Wiermer M, Stein M, et al. Pre- and postinvasion defenses both contribute to nonhost resistance in Arabidopsis. Science. 2005;310:1180-3.

52. Stein M, Dittgen J, Sanchez-Rodriguez C, Hou BH, Molina A, Schulze-Lefert P, et al. Arabidopsis PEN3/PDR8, an ATP binding cassette transporter, contributes to nonhost resistance to inappropriate pathogens that enter by direct penetration. Plant Cell. 2006;18:731-46.

53. Zhu S, Jeong R, Venugopal SC, Lapchyk L, Navarre D, Kachroo A, et al. SAG101 forms a ternary complex with EDS1 and PAD4 and is required for resistance signaling against turnip crinkle virus. PLoS Pathog. 2011;7:e1002318.

54. Wildermuth MC, Dewdney J, Wu G, Ausubel FM. Isochorismate synthase is required to synthesize salicylic acid for plant defence. Nature. 2001;414:562-5.

55. Zipfel C, Kunze G, Chinchilla D, Caniard A, Jones JDG, Boller T, et al. Perception of the bacterial PAMP EF-Tu by the receptor EFR restricts Agrobacterium-mediated transformation. Cell. 2006;125:749-60.

56. Gao M, Wang X, Wang D, Xu F, Ding X, Zhang Z, et al. Regulation of cell death and innate immunity by two receptor-like kinases in Arabidopsis. Cell Host Microbe. 2009;6:34-44

57. Pandey SP, Somssich IE. The role of WRKY transcription factors in plant immunity. Plant Physiol. 2009;150:1648-55.

58. Consonni C, Humphry ME, Hartmann HA, Livaja M, Durner J, Westphal L, et al. Conserved requirement for a plant host cell protein in powdery mildew pathogenesis. Nat Genet. 2006:38:716-20.

59. Arabidopsis Interactome Mapping Consortium. Evidence for network evolution in an Arabidopsis interactome map. Science. 2011;333:601-7.

60. Savard L, Li P, Strauss SH, Chase MW, Michaud M, Bousquet J. Chloroplast and nuclear gene sequences indicate late Pennsylvanian time for the last common ancestor of extant seed plants. Proc Natl Acad Sci U S A. 1994;91:5163-7.

61. Chaw S, Chang C, Chen H, Li W. Dating the monocot-dicot divergence and the origin of core eudicots using whole chloroplast genomes. J Mol Evol. 2004;58:424-41.

62. Wasiel AA, Rozeboom HJ, Hauke D, Baas B, Zandvoort E, Quax WJ, et al. Structural and functional characterization of a macrophage migration inhibitory factor homologue from the marine cyanobacterium Prochlorococcus marinus. Biochemistry. 2010;49:7572-81.

63. Chatterjee M, Borst $O$, Walker B, Fotinos A, Vogel S, Seizer $P$, et al. Macrophage migration inhibitory factor limits activation-induced apoptosis of platelets via CXCR7-dependent Akt signaling. Circ Res. 2014;115:939-49.

64. Rosengren E, Aman P, Thelin S, Hansson C, Ahlfors S, Björk P, et al. The macrophage migration inhibitory factor MIF is a phenylpyruvate tautomerase. FEBS Lett. 1997;417:85-8.

65. Orita M, Yamamoto S, Katayama N, Fujita S. Macrophage migration inhibitory factor and the discovery of tautomerase inhibitors. Curr Pharm Des. 2002:8:1297-317.

66. Kudrin A, Ray D. Cunning factor: macrophage migration inhibitory factor as a redox-regulated target. Immunol Cell Biol. 2008:86:232-8.

67. Dörmann P, Kim H, Ott T, Schulze-Lefert P, Trujillo M, Wewer V, et al. Cell-autonomous defense, re-organization and trafficking of membranes in plant-microbe interactions. New Phytol. 2014;204:815-22.

68. Panstruga R, Parker JE, Schulze-Lefert P. SnapShot. Plant immune response pathways. Cell. 2009;136:978-U6.

69. Nickel W, Rabouille C. Mechanisms of regulated unconventional protein secretion. Nat Rev Mol Cell Biol. 2009;10:148-55.

70. Weber C, Schober A, Zernecke A. Chemokines: key regulators of mononuclear cell recruitment in atherosclerotic vascular disease. Arterioscler Thromb Vasc Biol. 2004:24:1997-2008.

71. Ley K. Arrest chemokines. Microcirculation (New York, NY: 1994). 2003;10:289-95.

72. Segerer $S$, Johnson Z, Rek A, Baltus T, von Hundelshausen P, Kungl AJ, et al. The basic residue cluster (55)KKWVR(59) in CCL5 is required for in vivo biologic function. Mol Immunol. 2009;46:2533-8.

73. Liehn EA, Piccinini A, Koenen RR, Soehnlein O, Adage T, Fatu R, et al. A new monocyte chemotactic protein-1/chemokine CC motif ligand-2 competitor limiting neointima formation and myocardial ischemia/reperfusion injury in mice. J Am Coll Cardiol. 2010;56:1847-57.

74. Strüßmann T, Tillmann S, Wirtz T, Bucala R, von Hundelshausen P, Bernhagen J. Platelets are a previously unrecognised source of MIF. Thromb Haemost. 2013;110:1004-13.

75. Leng L, Metz CN, Fang Y, Xu J, Donnelly S, Baugh J, et al. MIF signal transduction initiated by binding to CD74. J exp med. 2003;197:1467-76
76. Tan TH, Edgerton SA, Kumari R, McAlister MS, Roe SM, Nagl S, et al. Macrophage migration inhibitory factor of the parasitic nematode Trichinella spiralis. Biochem J. 2001;357:373-83.

77. Soares AR, Marchiosi R, Siqueira-Soares, Rita de C, Barbosa de Lima R, Dantas dos Santos W, et al. The role of L-DOPA in plants. Plant Signal Behav. 2014;9:e28275

78. Solano F. Melanins: skin pigments and much more-types, structural models, biological functions, and formation routes. New J Sci. 2014;2014:1-28

79. Hu J, Baker A, Bartel B, Linka N, Mullen RT, Reumann S, et al. Plant peroxisomes: biogenesis and function. Plant Cell. 2012;24:2279-303.

80. Mitsuya S, El-Shami M, Sparkes IA, Charlton WL, Lousa, Carine De M, et al. Salt stress causes peroxisome proliferation, but inducing peroxisome proliferation does not improve $\mathrm{NaCl}$ tolerance in Arabidopsis thaliana. PLoS One. 2010:5:e9408.

81. Ortega-Galisteo AP, Rodríguez-Serrano M, Pazmiño DM, Gupta DK, Sandalio LM, Romero-Puertas MC. S-Nitrosylated proteins in pea (Pisum sativum L.) leaf peroxisomes: changes under abiotic stress. J Exp Bot. 2012;63:2089-103.

82. Shabab M. Role of plant peroxisomes in protection against herbivores. Subcell Biochem. 2013;69:315-28.

83. Sørhagen $\mathrm{K}$, Laxa $\mathrm{M}$, Peterhänsel $\mathrm{C}$, Reumann $\mathrm{S}$. The emerging role of photorespiration and non-photorespiratory peroxisomal metabolism in pathogen defence. Plant Biol. 2013;15:723-36.

84. Bednarek P, Piślewska-Bednarek M, Svatoš A, Schneider B, Doubský J Mansurova $M$, et al. A glucosinolate metabolism pathway in living plant cells mediates broad-spectrum antifungal defense. Science. 2009;323:101-6.

85. Jung $H$, Kim $T$, Chae $H Z$, Kim KT, Ha H. Regulation of macrophage migration inhibitory factor and thiol-specific antioxidant protein PAG by direct interaction. J Biol Chem. 2001;276:15504-10.

86. Kleemann R, Hausser A, Geiger G, Mischke R, Burger-Kentischer A, Flieger O, et al. Intracellular action of the cytokine MIF to modulate AP-1 activity and the cell cycle through Jab1. Nature. 2000;408:211-6.

87. Shen L, Hu J, Lu H, Wu M, Qin W, Wan D, et al. The apoptosis-associated protein BNIPL interacts with two cell proliferation-related proteins. MIF and GFER FEBS Lett. 2003;540:86-90.

88. Li Y, Lu C, Xing G, Zhu Y, He F. Macrophage migration inhibitory factor directly interacts with hepatopoietin and regulates the proliferation of hepatoma cell. Exp Cell Res. 2004;300:379-87.

89. Rodríguez-Trelles F, Tarrío R, Ayala FJ. Convergent neofunctionalization by positive Darwinian selection after ancient recurrent duplications of the xanthine dehydrogenase gene. Proc Natl Acad Sci U S A. 2003;100:13413-7.

90. Larkin MA, Blackshields G, Brown NP, Chenna R, McGettigan PA, McWilliam $\mathrm{H}$, et al. Clustal W and Clustal X version 2.0. Bioinformatics. 2007;23:2947-8.

91. Kelley LA, Sternberg, Michael JE. Protein structure prediction on the Web: a case study using the Phyre server. Nat Protoc. 2009:4:363-71.

92. Xu D, Zhang Y. Ab initio protein structure assembly using continuous structure fragments and optimized knowledge-based force field. Proteins. 2012;80:1715-35

93. Petersen TN, Brunak S, von Heijne G, Nielsen H. SignalP 4.0: discriminating signal peptides from transmembrane regions. Nat Methods. 2011;8:785-6.

94. Krogh A, Larsson B, von Heijne G, Sonnhammer EL. Predicting transmembrane protein topology with a hidden Markov model: application to complete genomes. J Mol Biol. 2001;305:567-80.

95. Claros MG, Vincens P. Computational method to predict mitochondrially imported proteins and their targeting sequences. Eur J Biochem. 1996:241:779-86.

96. Emanuelsson $\mathrm{O}$, Nielsen $\mathrm{H}$, von Heijne $\mathrm{G}$, Chloro $\mathrm{P}$. a neural network-based method for predicting chloroplast transit peptides and their cleavage sites. Protein Sci. 1999;8:978-84.

97. Nguyen B, Alex N, Pogoutse A, Provart N, Moses AM. NLStradamus: a simple Hidden Markov Model for nuclear localization signal prediction. BMC Bioinf. 2009;10:202.

98. La Cour T, Kiemer L, Mølgaard A, Gupta R, Skriver K, Brunak S. Analysis and prediction of leucine-rich nuclear export signals. Protein Eng Des Sel. 2004; 17:527-36.

99. Neuberger G, Maurer-Stroh S, Eisenhaber B, Hartig A, Eisenhaber F. Prediction of peroxisomal targeting signal 1 containing proteins from amino acid sequence. J Mol Biol. 2003;328:581-92.

100. Jones P, Binns D, Chang H, Fraser M, Li W, MCAnulla C, et al. InterProScan 5: genome-scale protein function classification. Bioinformatics. 2014;30:1236-40 
101. Winter D, Vinegar B, Nahal H, Ammar R, Wilson GV, Provart NJ. An "electronic fluorescent pictograph" browser for exploring anad analyzing large-scale biological data sets. PLoS One. 2007;2:e718.

102. Obayashi T, Kinoshita K. Coexpression landscape in ATTED-II: usage of gene list and gene network for various types of pathways. J Plant Res. 2010:123:311-9.

103. Usadel B, Nagel A, Steinhauser D, Gibon Y, Bläsing OE, Redestig H, et al. PageMan: an interactive ontology tool to generate, display, and annotate overview graphs for profiling experiments. BMC Bioin. 2006;7:535.

104. Tamura K, Peterson D, Peterson N, Stecher G, Nei M, Kumar S. MEGA5 molecular evolutionary genetics analysis using maximum likelihood, evolutionary distance, and maximum parsimony methods. Mol Biol Evol. 2011;28:2731-9.

105. Saitou N, Nei M. The neighbor-joining method: a new method for reconstructing phylogenetic trees. Mol Biol Evol. 1987;4:406-25.

106. Jones DT, Taylor WR, Thornton JM. The rapid generation of mutation data matrices from protein sequences. Comput Appl Biosci. 1992;8:275-82.

107. Felsenstein J. Confidence limits on phylogenies: an approach using the bootstrap. Evolution. 1985;39:783.

108. Nei M, Kumar S. Molecular evolution and phylogenetics. Oxford, New York: Oxford University Press; 2000

\section{Submit your next manuscript to BioMed Central and take full advantage of:}

- Convenient online submission

- Thorough peer review

- No space constraints or color figure charges

- Immediate publication on acceptance

- Inclusion in PubMed, CAS, Scopus and Google Scholar

- Research which is freely available for redistribution 